\title{
Noise Parameter Analysis of SiGe HBTs for Different Sizes in the Breakdown Region
}

\author{
Chie-In Lee, ${ }^{1,2}$ Yan-Ting Lin, ${ }^{1}$ and Wei-Cheng Lin ${ }^{1}$ \\ ${ }^{1}$ Department of Electrical Engineering, National Sun Yat-sen University, No. 70 Lienhai Rd., Kaohsiung 80424, Taiwan \\ ${ }^{2}$ Institute of Communications Engineering, National Sun Yat-sen University, No. 70 Lienhai Rd., Kaohsiung 80424, Taiwan \\ Correspondence should be addressed to Chie-In Lee; chiein@mail.ee.nsysu.edu.tw
}

Received 3 June 2016; Revised 9 September 2016; Accepted 18 September 2016

Academic Editor: Wei Wang

Copyright (c) 2016 Chie-In Lee et al. This is an open access article distributed under the Creative Commons Attribution License, which permits unrestricted use, distribution, and reproduction in any medium, provided the original work is properly cited.

\begin{abstract}
Noise parameters of silicon germanium ( $\mathrm{SiGe}$ ) heterojunction bipolar transistors (HBTs) for different sizes are investigated in the breakdown region for the first time. When the emitter length of SiGe HBTs shortens, minimum noise figure at breakdown decreases. In addition, narrower emitter width also decreases noise figure of SiGe HBTs in the avalanche region. Reduction of noise performance for smaller emitter length and width of SiGe HBTs at breakdown resulted from the lower noise spectral density resulting from the breakdown mechanism. Good agreement between experimental and simulated noise performance at breakdown is achieved for different sized SiGe HBTs. The presented analysis can benefit the RF circuits operating in the breakdown region.
\end{abstract}

\section{Introduction}

Characterization of high frequency noise for a transistor $[1,2]$ is essential to radio-frequency (RF) circuit designs because generated noise from transistors can determine the minimum signal strength that can be detected. Noise characteristics of a transistor can be dependent on device geometry $[3,4]$. There are some literatures $[3,4]$ that report geometry dependence of noise parameters for SiGe HBTs under the normal operating condition. RF circuits are usually prevented from operating in the high electric field region because noise performance can be degraded at breakdown. Nevertheless, high output power is demanded for some applications of voltage-controlled oscillators (VCOs) mentioned in [5-7]. Circuit designers can increase supply voltage of above collector-emitter breakdown voltage $\left(\mathrm{BV}_{\mathrm{CEO}}\right)$ for VCOs [8] to achieve high-power microwave source. For this $\mathrm{RF}$ circuit application in the impact ionization region, noise performance of SiGe HBTs needs to be considered because the breakdown mechanism can produce excess noise. In [9, 10], noise parameters of SiGe HBTs above $\mathrm{BV}_{\mathrm{CEO}}$ have been characterized. However, investigation of noise parameters of SiGe HBTs in the breakdown region has not been reported for different sizes.
Our previous research focused on breakdown noise modeling for SiGe HBTs [11]. In this work, we extend to the analysis of device geometry of noise parameters for SiGe HBTs in the avalanche region. Noise performance dependence on emitter size is reported in the impact ionization. Multiplication factors of SiGe HBTs are also analyzed for different sizes. This study with respect to device size can benefit the RF circuit applications in the breakdown region.

\section{Method}

Noise parameters of SiGe HBTs can be dependent on device geometry $[3,4]$. In order to extend geometry analysis from the normal operating region $[3,4]$ to the breakdown regime suitable for high-power VCO applications [5-7], we aim to study the emitter size dependence of noise parameters in the avalanche region in this work. This work utilizes the RF measurements to obtain the RF noise model for different sized devices and to analyze emitter size dependence of noise performance at breakdown. In [3], the DC measurements are employed to extract the equivalent circuit parameters of their noise model for geometry analysis in the normal operating region. On the other hand, for the breakdown operation, the literatures $[9,10]$ characterize the avalanche noise sources by 


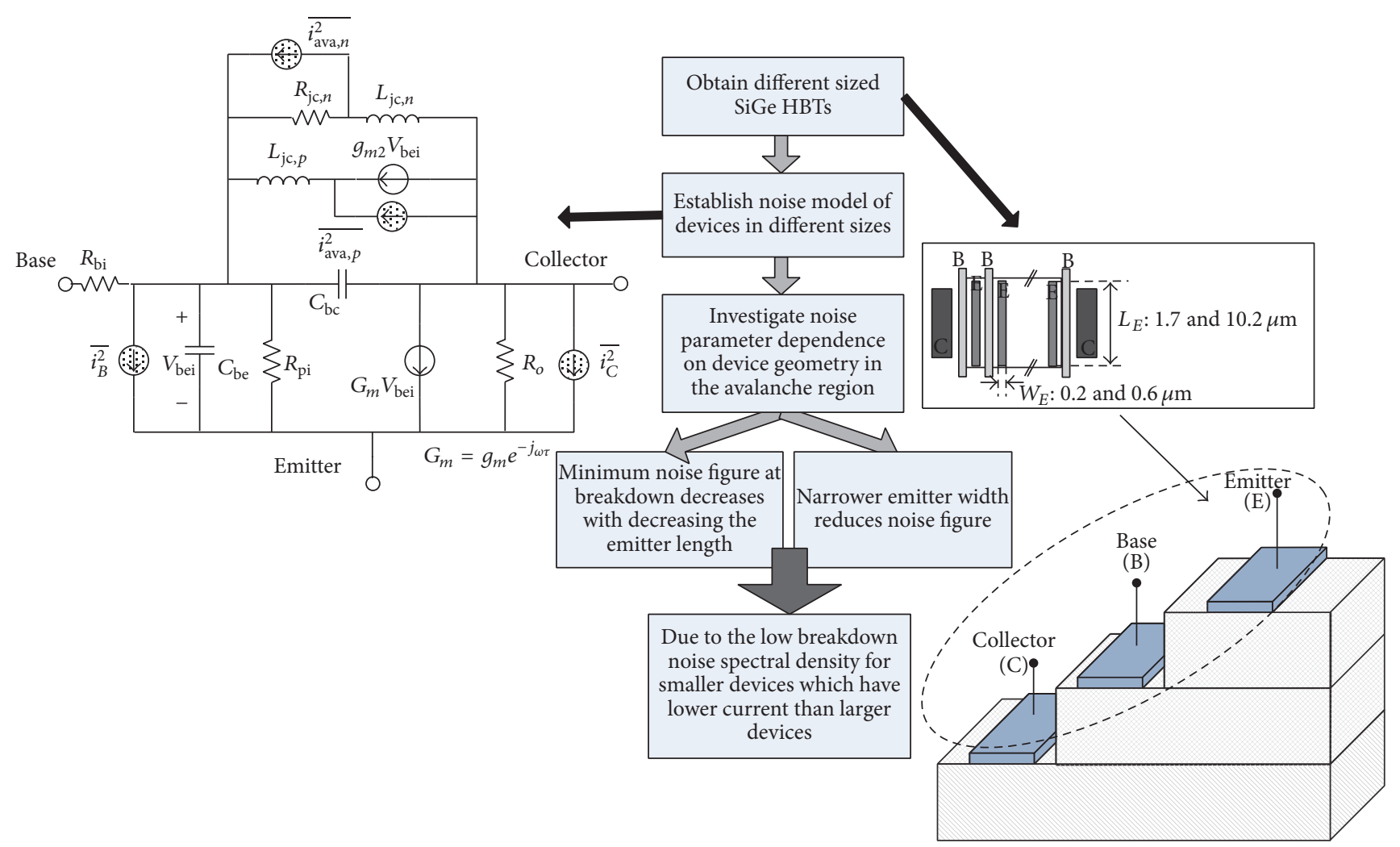

FIGURE 1: The flowchart of the presented analysis. The cross section of the SiGe HBTs with the diagram for different emitter lengths and emitter widths is also shown. The RF noise model including the inductive breakdown network is also presented. Although this noise model presented here for the sake of completeness is the same as [11], this work further extends to noise characterization for different sizes.

TABLE 1: Comparison between extracted and calculated breakdown noise spectral density at $V_{\mathrm{BE}}=1.1 \mathrm{~V}$ and $V_{\mathrm{CE}}=3.6 \mathrm{~V}$ for different sized SiGe HBTs.

\begin{tabular}{lcc}
\hline \multirow{2}{*}{ Operating region } & \multicolumn{2}{c}{ Characterization method } \\
& DC & RF \\
\hline Avalanche region & {$[9,10]^{*}$} & This work \\
Normal region & {$[3]$} & {$[3,4]$} \\
\hline
\end{tabular}

${ }^{*}$ Fixed device size.

using DC measurements for a fixed device size as shown in Table 1.

Different from the conventional characterization approach by the DC measurements, the presented RF measurement based method can be utilized to further reveal the RF phase delay of the breakdown mechanism. In the literature $[3,4]$, they also apply the RF measurements for noise characterization of SiGe HBTs. However, their analysis of noise parameters for different sized SiGe HBTs only focuses on the normal operating regime as shown in Table 1 . This work first studies the emitter size dependence of noise parameters in the avalanche region. The diagram of devices used in this study of noise parameter dependence on emitter size is shown in Figure 1. Similar to [12] where four different emitter sizes of transistors are employed, four different emitter sizes comprising $1.7 \times 0.2 \mu \mathrm{m}^{2}$ (total emitter area $=1.36 \mu \mathrm{m}^{2}$ and total emitter periphery $=15.2 \mu \mathrm{m}), 1.7 \times 0.6 \mu \mathrm{m}^{2}$ (total emitter area $=4.08 \mu \mathrm{m}^{2}$ and total emitter periphery $\left.=18.4 \mu \mathrm{m}\right), 10.2$ $\times 0.2 \mu \mathrm{m}^{2}$ (total emitter area $=8.16 \mu \mathrm{m}^{2}$ and total emitter periphery $=83.2 \mu \mathrm{m}$ ), and $10.2 \times 0.6 \mu \mathrm{m}^{2}$ (total emitter area $=24.48 \mu \mathrm{m}^{2}$ and total emitter periphery $=86.4 \mu \mathrm{m}$ ) with the same emitter finger of 4 are selected in this work in order to study noise performance under extreme conditions.

The selected geometry of devices is the allowable smallest and largest cases from the foundry design kit. The number of emitter fingers is fixed as 4 . The difference between this work and [12] is that we focus on noise parameter investigation at breakdown. In Figure 1, the SiGe HBT noise model for the presented investigation of noise parameter dependence on device size at breakdown is shown. Although the equivalent circuit model can be referred to [11], the description of each element is presented here for the sake of completeness. The avalanche noise sources $\overline{i_{\text {ava }, n}^{2}}$ and $\overline{i_{\text {ava }, p}^{2}}$ in the breakdown network are employed to describe the excess noise originated from random phenomena of the electron and hole avalanche multiplication mechanism, respectively. The noise spectral density is given as [11]

$$
S_{\mathrm{ibd}}=2 q I_{\mathrm{BC}} M^{2}\left[1-(1-k)\left(\frac{M-1}{M}\right)^{2}\right] .
$$

Furthermore, the electron breakdown inductance $L_{\mathrm{jc}, n}$ and resistance $R_{\mathrm{jc}, n}$ model the RF avalanche delay mechanism 


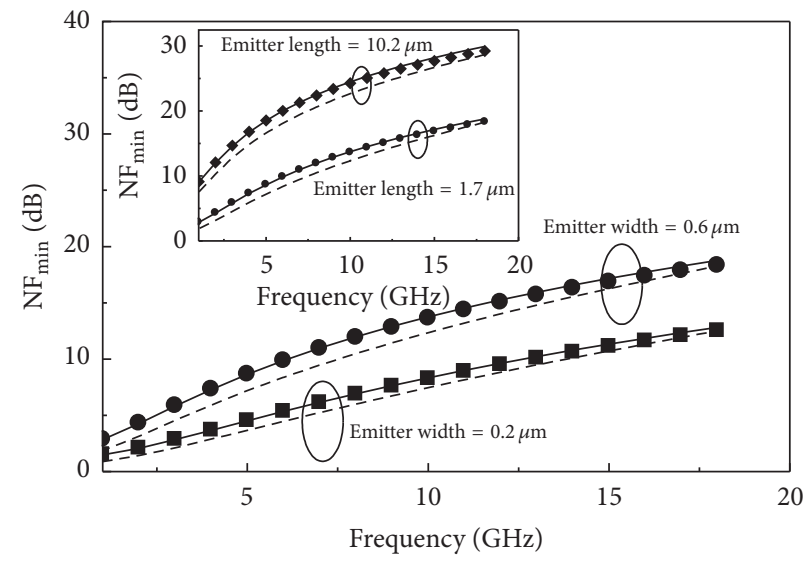

(a)

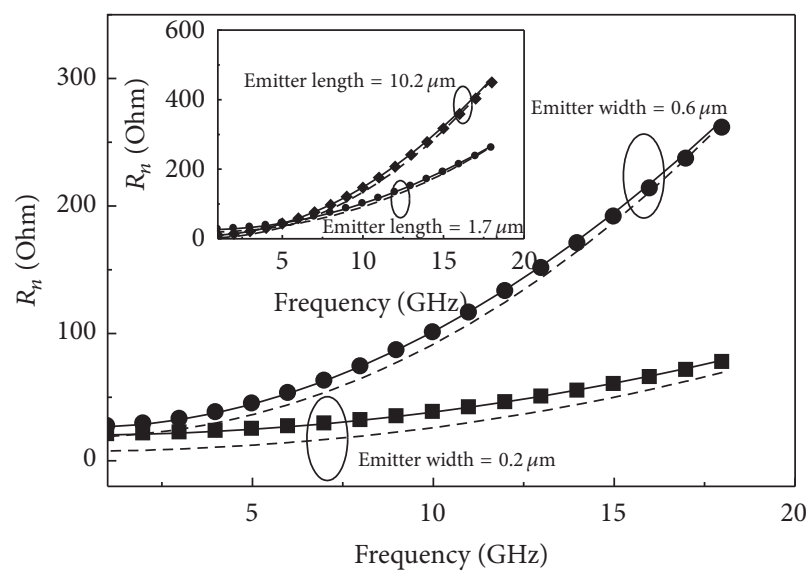

(b)

FIGURE 2: Measured (symbols) and simulated (lines) minimum noise figure and equivalent noise resistance at $V_{\mathrm{BE}}=1.1 \mathrm{~V}$ and $V_{\mathrm{CE}}=3.6 \mathrm{~V}$ in the breakdown region for different emitter widths. The emitter length is fixed as $1.7 \mu \mathrm{m}$. Solid and dash lines depict the results obtained from the noise model with and without considering the breakdown network, respectively. In the inset, the results for different emitter lengths as the emitter width is fixed as $0.6 \mu \mathrm{m}$ are shown. (a) Minimum noise figure. (b) Equivalent noise resistance.

of electrons. Reverse base current caused by holes [13] is described by the avalanche transconductance $g_{m 2}$ corresponding to hole conductance $R_{\mathrm{ic}, p}{ }^{-1}$ in the avalanche network. In addition, $L_{\mathrm{jc}, p}$ describes the RF avalanche delay mechanism of holes. $C_{\mathrm{be}}$ and $C_{\mathrm{bc}}$ represent the base-emitter and base-collector junction capacitance, respectively. $R_{\mathrm{pi}}$ is the base-emitter junction resistance. $G_{m}$ with time constant $\tau$ is the transconductance. $R_{o}$ is the output resistance. $R_{\mathrm{bi}}$ is the intrinsic base resistance. $\overline{i_{B}^{2}}$ and $\overline{i_{C}^{2}}$ represent the base current shot noise and collector current shot noise, respectively. Their noise correlation is also considered in the noise model. Equivalent circuit element and noise source determination of the presented RF noise model employ the procedure $[14,15]$ where the measured results at RF are fitted. The difference is that the RF avalanche network is further considered in this RF noise model in order to study the noise parameter dependence on emitter size in the impact ionization region.

\section{Results and Discussion}

SiGe HBTs with various emitter sizes were manufactured through the Taiwan Semiconductor Manufacturing Company by using the $0.18 \mu \mathrm{m}$ BiCMOS technology node. Noise parameters including the minimum noise figure $\mathrm{NF}_{\min }$, optimum source admittance $Y_{\mathrm{opt}}$, and equivalent noise resistance $R_{n}$ for different sized SiGe HBTs in the breakdown regime were measured through the ATN NP5B noise measurement system. The calibration procedure for the noise parameter measurement is performed from 1 to $18 \mathrm{GHz}$ by this ATN system as in [16]. In Figures 2 and 3, measured and simulated noise parameters are compared for SiGe HBTs with various emitter sizes, and good agreement is achieved, validating the presented RF noise model for different sizes.

In Figure 2(a), as the emitter length is fixed as $1.7 \mu \mathrm{m}$, $\mathrm{NF}_{\text {min }}$ decreases with decreasing emitter width from $0.6 \mu \mathrm{m}$ to $0.2 \mu \mathrm{m}$. In addition, equivalent noise resistance of SiGe

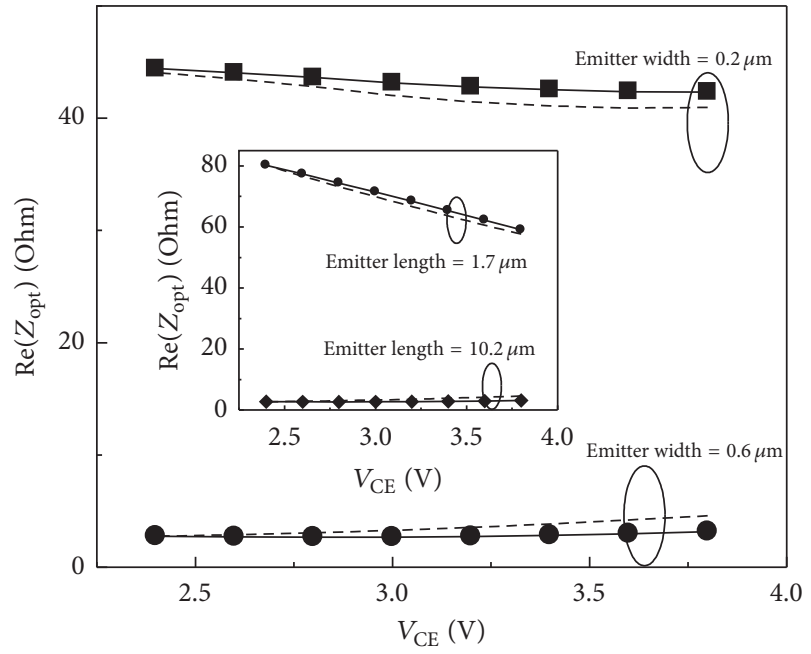

FIGURE 3: Measured (symbols) and simulated (lines) real part of optimal source impedance versus $V_{\mathrm{CE}}$ for different emitter widths. $V_{\mathrm{BE}}$ is at $1.1 \mathrm{~V}$. The operating frequency is at $2 \mathrm{GHz}$. The emitter length is fixed as $10.2 \mu \mathrm{m}$. Solid and dash lines depict the results obtained from the noise model with and without considering the breakdown network, respectively. In the inset, the results for different emitter lengths as the emitter width is fixed as $0.6 \mu \mathrm{m}$ are shown.

HBTs also decreases with reducing emitter width from $0.6 \mu \mathrm{m}$ to $0.2 \mu \mathrm{m}$ as shown in Figure 2(b). In Table 2, the determined breakdown noise spectral density is shown. $S_{\mathrm{ibd} n}$ and $S_{\mathrm{ibd} p}$ are avalanche noise spectral density resulting from $\overline{i_{\text {ava }, n}^{2}}$ and $\overline{i_{\text {ava }, p}^{2}}$, respectively. $S_{\mathrm{ib}}$ and $S_{\mathrm{ic}}$ are shot noise spectral density resulting from $\overline{i_{B}^{2}}$ and $\overline{i_{C}^{2}}$, respectively. As the emitter length is fixed as $1.7 \mu \mathrm{m}$, the spectral density decreases with decreasing emitter width. This lower $S_{\text {ibd }}$ reduces noise performance for the narrower emitter width $\mathrm{SiGe} \mathrm{HBT}$, and thus, $\mathrm{NF}_{\text {min }}$ 
TABLE 2: Comparison between extracted and calculated breakdown noise spectral density at $V_{\mathrm{BE}}=1.1 \mathrm{~V}$ and $V_{\mathrm{CE}}=3.6 \mathrm{~V}$ for different sized SiGe HBTs.

\begin{tabular}{lcc}
\hline & $\begin{array}{c}S_{\text {ibdn } n} / S_{\text {ibd } p \text { from }} \\
\text { calculation } \\
\left(\times 10^{-22} \mathrm{~A}^{2} \mathrm{~s}\right)\end{array}$ & $\begin{array}{c}S_{\mathrm{ibdn} n} / S_{\text {ibd } p \text { from }} \\
\text { presented noise } \\
\text { model }\left(\times 10^{-22} \mathrm{~A}^{2} \mathrm{~s}\right)\end{array}$ \\
\hline $0.2 \mu \mathrm{m} \times 1.7 \mu \mathrm{m}$ & $0.72 / 0.62$ & $0.69-0.74 / 0.60-0.65$ \\
$0.6 \mu \mathrm{m} \times 1.7 \mu \mathrm{m}$ & $0.80 / 0.70$ & $0.78-0.84 / 0.67-0.73$ \\
$0.2 \mu \mathrm{m} \times 10.2 \mu \mathrm{m}$ & $0.85 / 0.71$ & $0.83-0.89 / 0.69-0.74$ \\
$0.6 \mu \mathrm{m} \times 10.2 \mu \mathrm{m}$ & $0.92 / 0.75$ & $0.90-0.95 / 0.73-0.77$ \\
\hline
\end{tabular}

TABLE 3: The signal-to-noise ratio (SNR) of SiGe HBTs with different area at $V_{\mathrm{BE}}=1.1 \mathrm{~V}$ and $V_{\mathrm{CE}}=3.6 \mathrm{~V}$ in the breakdown region. The operating frequency is at $2 \mathrm{GHz}$.

\begin{tabular}{lcc}
\hline & $\begin{array}{c}\text { Emitter width } \\
=0.2 \mu \mathrm{m}\end{array}$ & $\begin{array}{c}\text { Emitter width } \\
=0.6 \mu \mathrm{m}\end{array}$ \\
\hline Emitter length $=1.7 \mu \mathrm{m}$ & $85.6 \mathrm{~dB}$ & $84.7 \mathrm{~dB}$ \\
Emitter length $=10.2 \mu \mathrm{m}$ & $85.2 \mathrm{~dB}$ & $78.6 \mathrm{~dB}$ \\
\hline
\end{tabular}

and $R_{n}$ become lower as shown in Figure 2. In addition, the signal-to-noise ratio (SNR) increases for the narrower emitter width as shown in Table 3 due to lower $S_{\text {ibd }}$ mentioned above. The high SNR represents low noise performance [17]. On the other hand, when the emitter width is fixed as $0.6 \mu \mathrm{m}, \mathrm{NF}_{\text {min }}$ and $R_{n}$ decrease and SNR increases with decreasing emitter length from $10.2 \mu \mathrm{m}$ to $1.7 \mu \mathrm{m}$ as shown in the inset of Figures 2(a) and 2(b) and Table 3 due to the reduced breakdown noise spectral density for the shorter emitter length shown in Table 2. The analysis of $\mathrm{NF}_{\min }$ and $R_{n}$ dependence on emitter length and width indicates that the smaller emitter size can reduce noise for $\mathrm{SiGe}$ HBTs operating in the breakdown region. This is explained as follows. The smaller emitter size can decrease the device current. As base-collector current of SiGe HBTs decreases, the noise spectral density can reduce [18]. Therefore, noise performance reduces with lowering the emitter size. Large $R_{n}$ can introduce difficulty of achieving minimum noise matching [19]. SiGe HBTs with narrow emitter width and short emitter length have smaller $R_{n}$ than the wide width and long length HBTs as shown in Figure 2(b). Therefore, small size of SiGe HBTs appears to be a way for obtaining the condition of minimum noise matching in low noise RF circuit designs.

The real part of optimal source impedance $\operatorname{Re}\left(Z_{\text {opt }}\right)$ shown in Figure 3 is used to investigate the geometry dependence of optimal source condition for noise matching of RF circuits as in [4]. Figure 3 shows that the narrower emitter width can increase $\operatorname{Re}\left(Z_{\text {opt }}\right)$. This is explained as follows. $\operatorname{Re}\left(Z_{\text {opt }}\right)$ can be proportional to the resistance seen at the input because the optimal source impedance is an input noise matching characteristic [4]. When the emitter width declines from $0.6 \mu \mathrm{m}$ to $0.2 \mu \mathrm{m}$, the breakdown resistance seen at the input increases from $98 \Omega$ to $126 \Omega$ for the fixed emitter length of $10.2 \mu \mathrm{m}$. This higher breakdown resistance seen at the input raises $\operatorname{Re}\left(Z_{\text {opt }}\right)$ for the narrower emitter width SiGe HBTs. On the other hand, when the emitter width is fixed as $0.6 \mu \mathrm{m}, \operatorname{Re}\left(Z_{\text {opt }}\right)$ increases with decreasing emitter length as shown in the inset of Figure 3 due to the higher breakdown resistance for the shorter emitter length $\mathrm{SiGe}$ HBTs. The breakdown resistance increases from $98 \Omega$ to $156 \Omega$ for emitter length decreased from $10.2 \mu \mathrm{m}$ to $1.7 \mu \mathrm{m}$. Conventional analysis for the real part of optimal source impedance for different device sizes focuses on the normal operating region [4]. This work further investigates the real part of optimal source impedance dependence on device size in the avalanche region. This analysis demonstrates that the smaller emitter size can increase real part of optimal source impedance in the avalanche region due to increased breakdown resistance which can influence the input noise matching characteristic.

The simulated results by using the conventional noise model without considering the presented RF avalanche network are compared in the impact ionization region and a deviation is found for different sized SiGe HBTs as shown in Figures 2 and 3. Larger deviation of $S_{\mathrm{ib}}$ and $S_{\mathrm{ic}}$ in the conventional model is observed for the longer emitter length and wider emitter width sized devices as shown in Table 4 because the breakdown noise spectral density is more pronounced for large sized SiGe HBTs as reported in Table 2. Instead, the obtained $S_{\mathrm{ib}}$ and $S_{\mathrm{ic}}$ from our RF noise model are close to the calculated values. In addition, the extracted breakdown noise spectral density can agree well with the theoretical results using (1) for different sized SiGe HBTs.

\section{Conclusions}

In this paper, the investigation of noise parameter dependence on device geometry for SiGe HBTs operating in the avalanche region is presented for the first time. SiGe HBT minimum noise figure at breakdown decreases with decreasing the emitter length. In addition, narrower emitter width reduces noise figure in the avalanche region. Decreased noise with decreasing device size at breakdown is found to be due to the low breakdown noise spectral density for smaller devices which have lower current than larger devices. Good agreement between experiment and simulation results of noise performance at breakdown is obtained for different sized SiGe HBTs. This study with respect to device size can benefit the RF circuit applications in the breakdown region.

\section{Competing Interests}

The authors declare that they have no competing interests.

\section{Acknowledgments}

The authors would like to thank National Chip Implementation Center (CIC), Hsinchu, Taiwan, for chip support, the Taiwan Semiconductor Manufacturing Company (TSMC), Hsinchu, Taiwan, for chip fabrication, the National Nano Device Laboratories (NDL), Hsinchu, Taiwan, for the high frequency noise measurement support, and the Wireless 
TABLE 4: Determined base and collector noise spectral density for different sized SiGe HBTs in the breakdown region of $V_{\mathrm{BE}}=1.1 \mathrm{~V}$ and $V_{\mathrm{CE}}=3.6 \mathrm{~V}$. The results obtained from the presented noise model, calculation, and conventional model are compared. The variation is indicated for the results obtained from the presented model as the deviation of noise performance fitting is within $1 \%$.

\begin{tabular}{lccc}
\hline & $\begin{array}{c}S_{\mathrm{ib}} / S_{\mathrm{ic}} \text { from presented } \\
\text { noise model } \\
\left(\times 10^{-24} \mathrm{~A}^{2} \mathrm{~s}\right)\end{array}$ & $\begin{array}{c}S_{\mathrm{ib}} / S_{\mathrm{ic}} \text { from calculation } \\
\left(\times 10^{-24} \mathrm{~A}^{2} \mathrm{~s}\right)\end{array}$ & $\begin{array}{c}S_{\mathrm{ib}} / S_{\mathrm{ic}} \text { from conventional noise model } \\
\left(\times 10^{-24} \mathrm{~A}^{2} \mathrm{~s}\right)^{*}\end{array}$ \\
\hline $0.2 \mu \mathrm{m} \times 1.7 \mu \mathrm{m}$ & $5.44-5.97 / 42.83-43.32$ & $5.64 / 43.11$ & $6.11 / 43.36$ \\
$0.6 \mu \mathrm{m} \times 1.7 \mu \mathrm{m}$ & $7.21-7.76 / 46.3-47.0$ & $7.41 / 46.76$ & $8.01 / 47.33$ \\
$0.2 \mu \mathrm{m} \times 10.2 \mu \mathrm{m}$ & $8.54-8.90 / 48.87-49.36$ & $8.86 / 49.12$ & $9.41 / 49.89$ \\
$0.6 \mu \mathrm{m} \times 10.2 \mu \mathrm{m}$ & $11.01-11.72 / 55.68-56.19$ & $11.65 / 55.89$ & $12.87 / 56.93$ \\
\hline
\end{tabular}

* It is difficult to achieve error below $1 \%$ by the conventional noise model so that the determined values deviate from calculation results.

Communication Antenna Research Center, Kaohsiung, Taiwan, for the support.

\section{References}

[1] J.-D. Chen, "A low-power ultrawideband low-noise amplifier in $0.18 \mu \mathrm{m}$ CMOS technology," Active and Passive Electronic Components, vol. 2013, Article ID 953498, 10 pages, 2013.

[2] A. Caddemi and M. Sannino, "Small-signal and noise model determination for double polysilicon self-aligned bipolar transistors," Active and Passive Electronic Components, vol. 17, no. 3, pp. 167-175, 1994.

[3] W.-K. Lee, S. Lam, and M. Chan, "Effects of spacer thickness on noise performance of bipolar transistors," IEEE Transactions on Electron Devices, vol. 51, no. 9, pp. 1534-1537, 2004.

[4] S. Zhang, G. Niu, J. D. Cressler, A. J. Joseph, G. Freeman, and D. L. Harame, "The effects of geometrical scaling on the frequency response and noise performance of SiGe HBTs," IEEE Transactions on Electron Devices, vol. 49, no. 3, pp. 429-435, 2002.

[5] H. Liu, X. Zhu, C. C. Boon, and X. Yi, "Design of an oscillator with low phase noise and medium output power in a 0.25 $\mu \mathrm{m} \mathrm{GaN-on-SiC} \mathrm{high} \mathrm{electron-mobility} \mathrm{transistors} \mathrm{technology,"}$ IET Microwaves, Antennas \& Propagation, vol. 9, no. 8, pp. 795801, 2015.

[6] H. Liu, X. Zhu, C. C. Boon, X. Yi, M. Mao, and W. Yang, "Design of ultra-low phase noise and high power integrated oscillator in $0.25 \mu \mathrm{m} \mathrm{GaN}$-on-SiC HEMT technology," IEEE Microwave and Wireless Components Letters, vol. 24, no. 2, pp. 120-122, 2014.

[7] W. Jiang, N. Shimada, S. D. Prasad, and K. Yatsui, "Experimental and simulation studies of new configuration of virtual cathode oscillator," IEEE Transactions on Plasma Science, vol. 32, no. 1, pp. 54-59, 2004.

[8] H. Li, H.-M. Rein, T. Suttorp, and J. Böck, "Fully integrated SiGe VCOs with powerful output buffer for 77-GHz automotive radar systems and applications around $100 \mathrm{GHz}$," IEEE Journal of Solid-State Circuits, vol. 39, no. 10, pp. 1650-1658, 2004.

[9] P. Sakalas, M. Ramonas, M. Schroter, C. Jungemann, A. Shimukovitch, and W. Kraus, "Impact ionization noise in SiGe HBTs: comparison of device and compact modeling with experimental results," IEEE Transactions on Electron Devices, vol. 56, no. 2, pp. 328-336, 2009.

[10] J. C. J. Paasschens and R. De Kort, "Modelling the excess noise due to avalanche multiplication in (hetero-junction) bipolar transistors," in Proceedings of the IEEE Bipolar/BiCMOS Circuits and Technology Meeting, pp. 108-111, September 2004.
[11] C. I. Lee, Y. T. Lin, and W. C. Lin, "An improved noise model for SiGe HBT with an inductive breakdown network in the avalanche region," IEEE Transactions on Device and Materials Reliability, vol. 15, no. 4, pp. 588-594, 2015.

[12] R. J. Malik, L. M. Lunardi, R. W. Ryan, S. C. Shunk, and M. D. Feuer, "Submicron scaling of AlGaAs/GaAs self-aligned thin emitter heterojunction bipolar transistors (SATE-HBT) with current gain independent of emitter area," Electronics Letters, vol. 25, no. 17, pp. 1175-1177, 1989.

[13] C.-I. Lee, Y.-T. Lin, and W.-C. Lin, "Investigation of linearity in the high electric field region for SiGe HBTs based on Volterra series," IEEE Transactions on Device and Materials Reliability, vol. 14, no. 4, pp. 1049-1055, 2014.

[14] J. P. Roux, L. Escotte, R. Plana, J. Graffeuil, S. L. Delage, and H. Blanck, "Small-signal and noise model extraction technique for heterojunction bipolar transistor at microwave frequencies," IEEE Transactions on Microwave Theory and Techniques, vol. 43, no. 2, pp. 293-298, 1995.

[15] L. Escotte, J.-P. Roux, R. Plana, J. Graffeuil, and A. Gruhle, "Noise modeling of microwave heterojunction bipolar transistors," IEEE Transactions on Electron Devices, vol. 42, no. 5, pp. 883-889, 1995.

[16] S. Lee, K. J. Webb, V. Tilak, and L. F. Eastman, "Intrinsic noise equivalent-circuit parameters for AlGaN/GaN HEMTs," IEEE Transactions on Microwave Theory and Techniques, vol. 51, no. 5, pp. 1567-1577, 2003.

[17] D. M. Pozar, Microwave Engineering, Wiley, New York, NY, USA, 3rd edition, 2005.

[18] R. J. McIntyre, "Multiplication noise in uniform avalanche diodes," IEEE Transactions on Electron Devices, vol. 13, no. 1, pp. 164-168, 1966.

[19] G. Dambrine and J.-P. Raskin, "High-frequency four noise parameters of silicon-on-insulator-based technology MOSFET for the design of low-noise RF integrated circuits," IEEE Transactions on Electron Devices, vol. 46, no. 8, pp. 1733-1741, 1999. 


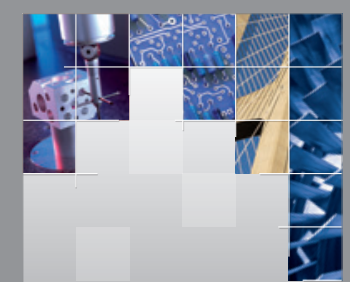

\section{Enfincering}
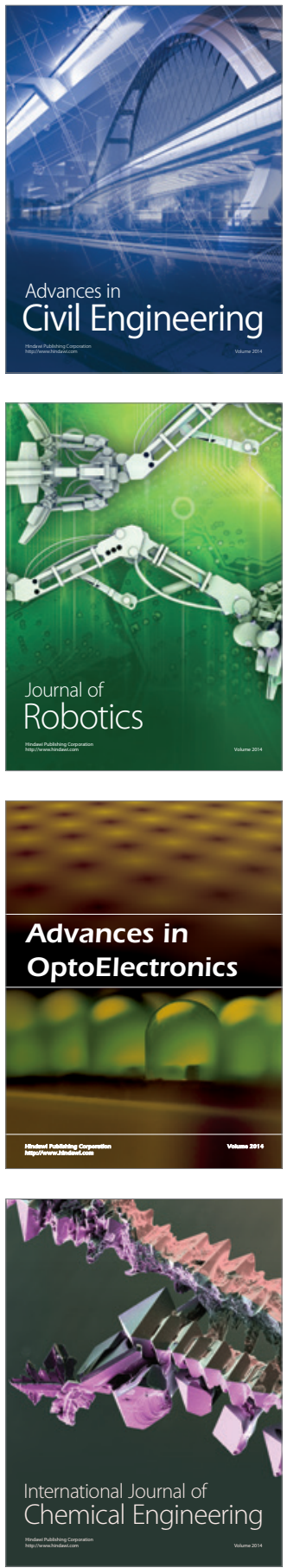

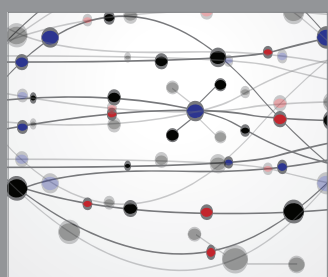

The Scientific World Journal

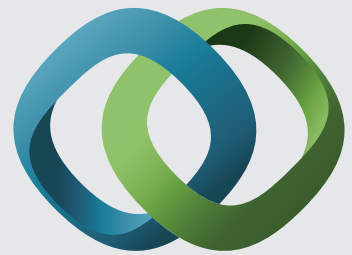

\section{Hindawi}

Submit your manuscripts at

http://www.hindawi.com
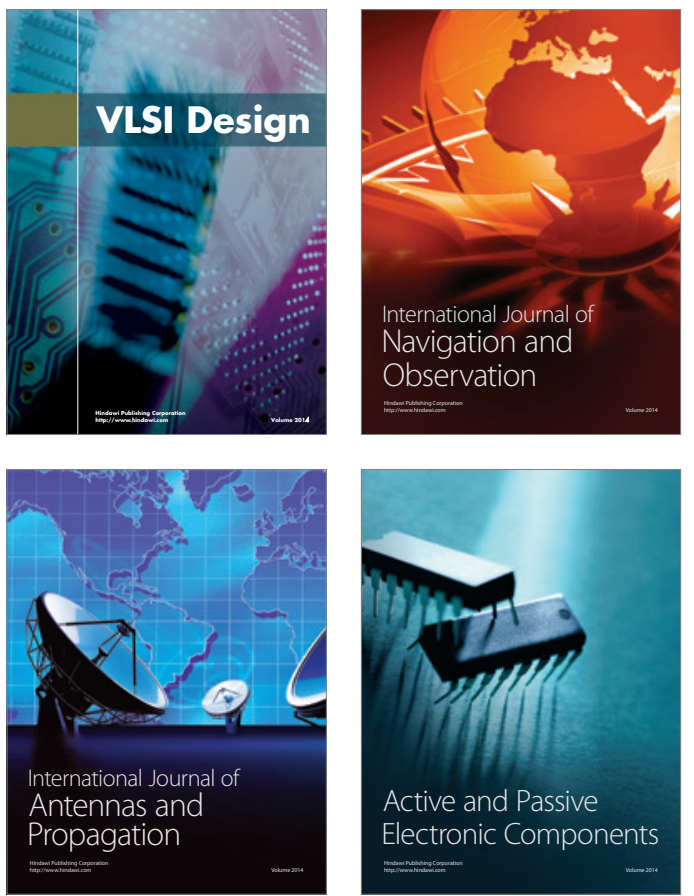
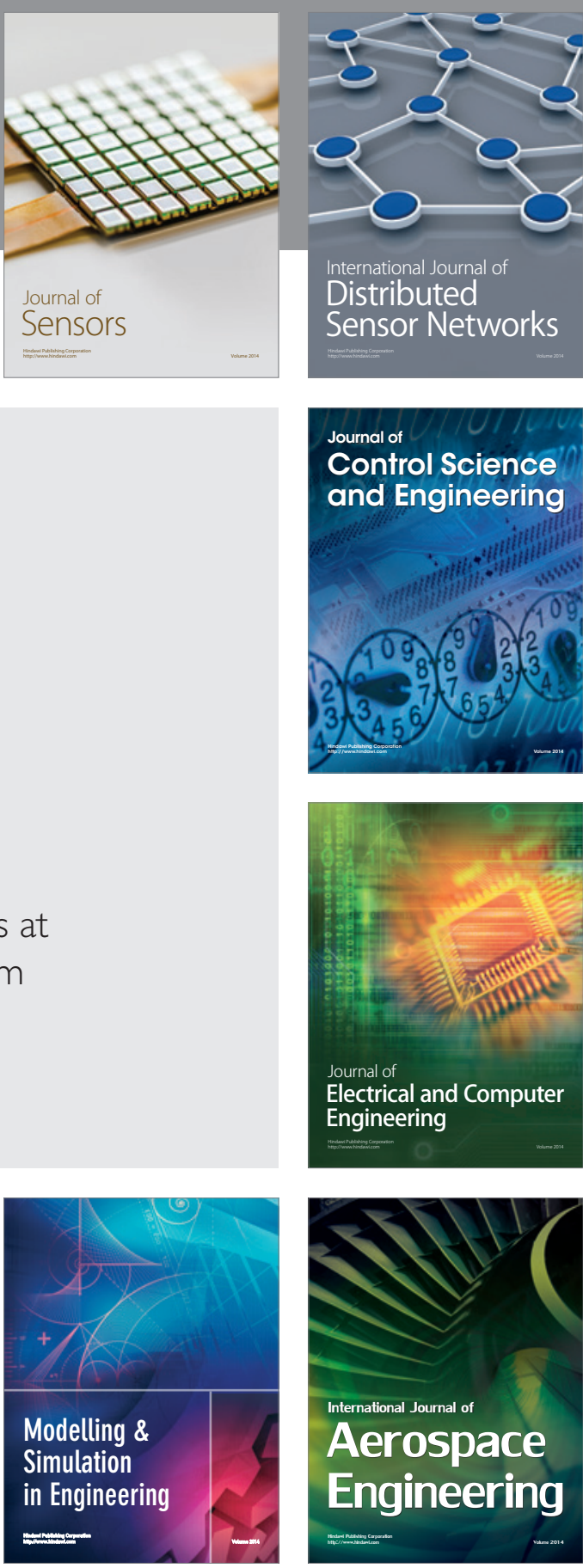

International Journal of

Distributed

Sensor Networks

Journal of

Control Science

and Engineering
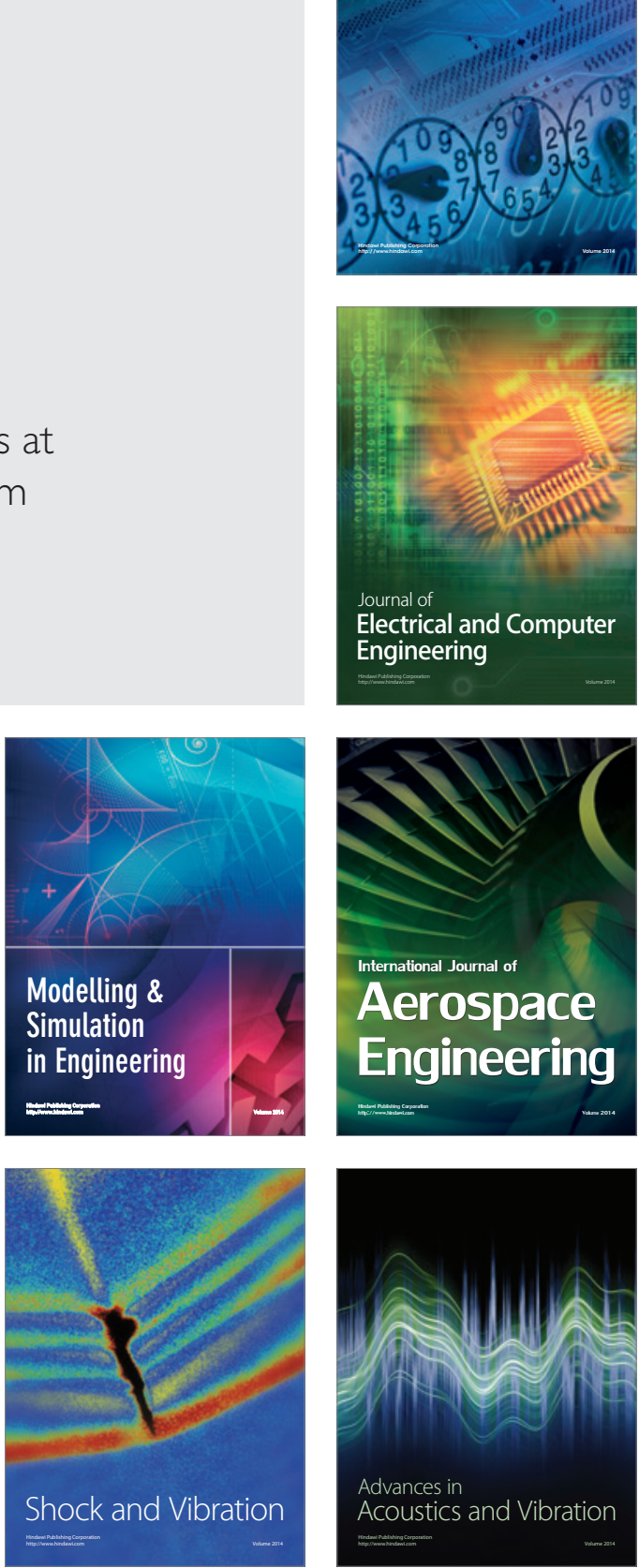